\title{
A Modal Logic Framework for Human-Computer Spoken Interaction
}

\author{
L. Villaseñor-Pineda ${ }^{1}$, M. Montes-y-Gómez ${ }^{1}$ and J. Caelen ${ }^{2}$ \\ ${ }^{1}$ Instituto Nacional de Astrofísica, Óptica y Electrónica (INAOE), Mexico. \\ \{villasen, mmontesg\} @inaoep.mx \\ ${ }^{2}$ Laboratoire CLIPS-IMAG, Université Joseph Fourier, France. \\ jean.caelendimag. fr
}

\begin{abstract}
One major goal of human computer interfaces is to simplify the communication task. Traditionally, users have been restricted to the language of computers for this task. With the emerging of the graphical and multimodal interfaces the effort required for working with a computer is decreasing. However, the problem of communication is still present, and users continue caring about the communication task when they deal with a computer. Our work focuses on improving the communication between the human and the computer. This paper presents the foundations of a multimodal dialog model based on a modal logic, which integrates the speech and the action under the same framework.
\end{abstract}

Keywords: human computer spoken interaction, speech acts, multimodal interaction, and modal logic.

\section{Introduction}

The first dialog systems used the speech as the unique communication channel. How ever, the human communication is strongly multimodal. The lips movement, the facial expressions, and the gestures are all of them key elements in the human interchange of information.

Current multimodal dialog systems attempt to integrate several communication modalities along with the speech. The construction of this kind of systems is a complex task [7, 9, 12]. It considers several problems such as: speech recognition, natural language understanding, knowledge representation, fusion of the different input modalities in a coherent message, the definition of a dialog model and ot hers.

This paper focuses on the definition of a dialog model. It presents the foundations of a multimodal dialog model based on a modal logic, which represent the rules of the conversation and integrates in the same framework the direct actions (those accomplished with a device of direct designation such as the mouse) and the spoken ones (those orally requested by the user to the machine). This consideration is of great relevance because the spoken actions are not performed immediately such as the direct ones. Thus, the evolution of a spoken action must be controlled during the dialog: from the moment it is proposed until the time it is satisfied.

The proposed model is supported by the theory of speech acts [1,11]. It is based on the hypothesis that the dialog is conduced by the metal states that maintain the beliefs, desires and intentions of the user. Nevertheless, this model does not atempt to set a 
human behavior to the machine, but only to give it the logical elements to hold its actions [2]. The idea of treating the speech as an action is not original (see for instance $[3,4,8]$ ), however our logic implements a general mechanism in which the spoken action is controlled over all the dialogic interchange. This way, it models the convergence of a cooperative dialog [17].

The paper is organized as follows. Section 2 presents the complete logic framework. Section 3 shows a short but illustrative example of a dialog conduced by the proposed logic. Finally, section 4 discusses our conclusions and future work.

\section{A logic for the dialog}

This section presents the basis of a logic that models the information interchange between a user and a machine. This logic, inspired by previous works $[2,3,6,10,13$, $14,15,16]$, proposes the integration of the dialog acts in a framework based on the action. It contains elements of an epistemological logic -to represent the knowledge-, a dynamic logic - to describe the action and its effects - and a dialogical logic -to represent the obligations and intentions expressed during the dialog.

\subsection{Basic concepts}

This subsection defines the three basic concepts of our logic: knowledge, action and intention.

The knowledge is represented by the operator $s$ (to know). For instance, the formula $U s \varphi$ expresses that the user knows the proposition $\varphi$ (to make the distinction between the user and the machine, the two possible agents of our logic, we used the letter $U$ for the user and $M$ for the machine).

In order to represent the action, we introduce the notion of an event. An event $U f \alpha$ is the achievement of the action $\alpha$ by the user (or $M f \alpha$ in the case of the machine), which has the proposition $\varphi$ as result. Using the notation of a dynamic logic we have the following formula $[U f \alpha] \varphi$. This formula indicates that after the execution of $\alpha$ by the user, $\varphi$ is true. An action can be a base action, i.e., an elemental instruction, or even a task, i.e., a sequence of actions organized by a plan.

The intention is represented by the operator $i$. Only the user is capable of having intentions, thus the formula $U i$ pexpresses the intention of the user to make $\varphi$ true.

\subsection{Dialog acts}

A dialog act is an action causing a change in, on one hand, the task or the machine knowledge about the task, and on the other hand, the dialog itself. Hence, a dialog act is defined as an event $[U f \alpha]\left(\varphi_{\alpha} \wedge \varphi_{d}\right)$, where the result is the set of changes related with the task $\varphi_{\alpha}$ and with the dialog $\varphi_{d}$

The changes related with the task are the own effects of the action $\left(\varphi_{\alpha}\right)$, while the changes related with the dialog $\varphi_{d}$ shows the progress in the dialog state after each interchange of information. In our case, the goal of any dialog is to complete the task intended by the user. This way, each dialog act produces an effect over the goal $\left(\varphi_{d}\right)$. Further, the effects of a spoken action depend on the comprehension of the action by the receptor (who may not understand or even perform an erroneous operation), and 
consequently, they are not always predictable. In other words, the effects of a spoken action only make sense when they are related with the intentions of the speaker. Thus, the evolution of a spoken action must be controlled during the dialog: from the moment it is proposed until the time it is satisfied. For this reason we define the following states for an action:

? Proposed goal; an action to be perform it.

$+\quad$ Reached goal; an accomplished action with no confirmation.

+ Satisfied goal; a completed (and correct) action.

@ Aborted goal.

The dialog acts are expressed, by means of direct and spoken actions, as follows:

$U f \alpha, M f \alpha \quad$ the user or the machine performs $\alpha$.

Uff $\alpha, M f f \alpha \quad$ the user asks the machine to perform the action $\alpha$ (or vice versa)

$U f s \phi, M f s \phi \quad$ the user informs $\phi$ to the machine (or vice versa)

Uffs $\phi, M f f s \phi \quad$ the user asks the machine to inform $\phi$ (or vice versa)

where the action $f_{s}$ is an abbreviation of the base action to share. Ufs $\phi \equiv$ Uf share $\phi$

\subsection{Definition of the Language $L_{d}$}

Definition 1. If $\mathrm{T}$ is the set of propositional symbols, $A b$ a finite set of base actions, $U$ the symbol to named the user and $M$ the machine, then the language $L_{d}$ is defined as follows:

$$
\begin{aligned}
& L_{d} \text { is the smallest subset of } T \text { such that: } \\
& \text { - if } \varphi, \psi \in L_{d} \text { then } \neg \varphi, \varphi \vee \psi \in L_{d} \\
& \text { - if } \alpha \in A c, \text { y } \varphi \in L_{d} \text { then } \\
& \text { - } \quad U s \varphi, M s \varphi,[U f \alpha] \varphi,[M f \alpha] \varphi, U i \varphi \in L_{d}
\end{aligned}
$$

where $A c$ is the smallest subset of $A b$, such that:

- if $\alpha \in A b$ then $\alpha \in A c$

- if $\varphi \in L_{d}$ then verify $(\varphi) \in A c$

- if $\alpha \in A c$ and $\beta \in A c$ then $\alpha ; \beta \in A c$

We use the abbreviated notations $\varphi \wedge \psi$ for $\neg(\neg \varphi \vee \neg \psi)$ and $\varphi \supset \psi$ for $\neg(\varphi \wedge \neg \psi)$. The true abbreviation is considered as a valid formula, e.g. $\varphi \vee \neg \varphi$, and false as an abbreviation of $\neg$ true.

Definition 2. The semantic of the language $L_{d}$ The class $\mathbf{M}$ of the models of Kripke contains all the tuples $M=\left\langle S, \pi, R_{U}, R_{M}, I_{U}, r_{U}, r_{M}>\right.$ such that:

i) $\quad S$ is the set of possible worlds, or states.

ii) $\pi$ is a function that assigns truth values to the propositional symbols of $T$ in a possible state $s(\pi(s): T \rightarrow\{1,0\}$ for all $s \in S)$. 
iii) $R_{U}$ is a binary relation among the possible states of $S$. It is the relation of accessibility to the user knowledge ( $R_{U} \subseteq S \times S$ )

iv) $R_{M}$ is a binary relation among the possible states of $S$. It is the relation of accessibility to the machine knowledge ( $R_{M} \subseteq S \times S$ )

v) $\quad I_{U}$ is a binary relation among the possible states of $S$. It is the relation of accessibility to the user intentions ( $I_{U} \subseteq S \times S$ )

vi) $r_{U}$ is a relation among the set of possible states caused by the accomplishment of the action $\alpha$ by the user in a possible state $s,\left(r_{U}: A c \times S \rightarrow \wp(S)\right)$

vii) $r_{M}$ is a relation among the set of possible states caused by the accomplishment of the action $\alpha$ by the machine in a possible state $s,\left(r_{M}: A c \times S \rightarrow \wp(S)\right)$

Definition 3. Let $M=\left\langle S, \pi, R_{U}, R_{M}, I_{U}, r_{U}, r_{M}\right\rangle$ be a Kripke model of class $\mathbf{M}$. The truth value of a proposition ( $\mid=)$ in a possible state $s$, based on the model $M$, is inductively defined as follows:

$$
\begin{array}{ll}
M, s \mid=\varphi & \text { iff } \pi(s)(\varphi)=1 \text { for } \varphi \in T \\
M, s \mid=\neg \varphi & \text { iff } M, s \mid \neq \varphi \\
M, s \mid=\varphi \vee \phi & \text { iff } M, s^{\prime} \mid=\varphi \text { or } M, s \mid=\phi \\
M, s \mid=[U f \alpha] \varphi & \text { iff } \forall s^{\prime}\left[s^{\prime} \in r_{U}(\alpha, s) \Rightarrow M, s^{\prime} \mid=\varphi\right] \\
M, s \mid=[M f \alpha] \varphi & \text { iff } \forall s^{\prime}\left[s^{\prime} \in r_{M}(\alpha, s) \Rightarrow M, s^{\prime} \mid=\varphi\right] \\
M, s \mid=U s \varphi & \text { iff } \forall s^{\prime}\left[\left(s, s^{\prime}\right) \in R_{U} \Rightarrow M, s^{\prime} \mid=\varphi\right] \\
M, s \mid=M s \varphi & \text { iff } \forall s^{\prime}\left[\left(s, s^{\prime}\right) \in R_{M} \Rightarrow M, s^{\prime} \mid=\varphi\right] \\
M, s \mid=U i \varphi & \text { iff } \forall s^{\prime}\left[\left(s, s^{\prime}\right) \in I_{U} \Rightarrow M, s^{\prime} \mid=\varphi\right]
\end{array}
$$

where $r_{U}$ and $r_{M}$, denoted as $r_{A}$ for their equivalence and shake of simplicity, are defined by:

$$
\begin{array}{ll}
r_{A}(\operatorname{verify}(\varphi), s) & =\{s\} \text { if } M, s \mid=\varphi \\
& =\varnothing \text { in other case } \\
r_{A}((\alpha ; \beta), s) & =r_{A}\left(\beta, r_{A}(\alpha, s)\right)
\end{array}
$$

\subsection{Definition of the axioms}

This subsection presents the main axioms of our logic. The first part describes the axioms about the knowledge, the second part introduces some concepts related with the goal evolution, and the third part explains the axioms about the cooperative dia$\log$.

\subsubsection{Knowledge characterization}

The following axioms describe the machine and the user knowledge, and for the case of the machine, they characterize it knowledge about the user intentions.

Let $A=\{U, M\}$ :

(A1) As $\varphi \wedge \mathrm{A} s(\varphi \supset \phi) \supset$ As $\phi \quad$ axiom $K$

(A2) As $\varphi \supset \varphi$

The user and the machine just know true facts

(A3) As $\varphi \supset \mathrm{A} s \mathrm{~A} s \varphi$ positive introspection 
The user and the machine know the facts that they know

(A4) $\neg \mathrm{A} s \varphi \mathrm{A} s \neg \mathrm{A} s \varphi \quad$ negative introspection

The user and the machine know the facts they do not know

\subsubsection{Goal evolution}

In our model, the structure of the dialog is based on the user intention. This intention is expresses as an action (or plan), which its effect is the desired final state. Thus, the realization of a dialog act generally produces a movement to the goal. This movement depends on the current situation and in the dialog act. It is represented by three states: (i) a proposed goal, when the user orders the machine the execution of an action $(U f f)$, or asks it for an information (Uffs); (ii) a reached goal, when the machine responds (Mfs), or execute the requested action (Mf); (iii) finally, a satisfied goal, when the user is pleased with the machine response. Of course, the user can abort a goal at any moment. The following paragraphs describe this evolution at detail.

\section{The user asks the machine to perform an action}

i) A proposed goal is the effect of a user request. It is expressed as a user intention (that the machine performs some action) integrated with the machine knowledge.

$[U f f \alpha](M s U i[M f \alpha] \varphi) \equiv[U f f \alpha](?[M f \alpha] \varphi)$

Here the abbreviation? $M f \alpha$ designates the action $M f \alpha$ as a proposed goal.

ii) A reached goal emerges when the action requested by the user becom es true.

$$
\begin{array}{rl}
M s & U i[M f \alpha] \varphi \wedge[M f \alpha] \varphi \\
& \equiv ?[M f \alpha] \varphi \wedge[M f \alpha] \varphi \\
& \equiv+[M f \alpha] \varphi
\end{array}
$$

where the abbreviation $+M f \alpha$ designates the action $M f \alpha$ as a reached goal.

iii) A satisfied goal materializes when the user admits the action of the machine as acceptable. This acceptation can be explicit (when the user informs that his intention is no more related with the propos ed action), or even implicit (when the user asks the machine to perform a different action -not related with the previous one).

$$
\begin{array}{rl}
M s & U i[M f \alpha] \varphi \wedge([U f s U \neg i[M f \alpha] \varphi] \phi \vee([U f f \beta] \gamma \wedge \neg \operatorname{rel}(\varphi, \beta))) \\
& \equiv ?[M f \alpha] \varphi \wedge([U f s U \neg \mathrm{i}[M f \alpha] \varphi] \phi \vee([U f f \beta] \gamma \wedge \neg \operatorname{rel}(\varphi, \beta))) \\
& \equiv++[M f \alpha] \varphi
\end{array}
$$

Here, the abbreviation $++M f \alpha$ designates the action $M f \alpha$ as a satisfied goal.

iv) An aborted goal occurs when the user informs the machine that his desire is no more the achievement of the action in progress.

$$
\begin{array}{rl}
M s & U i[M f \alpha] \varphi \wedge(\text { Ufs } U i[M \neg f \alpha] \varphi) \\
& \equiv ?[M f \alpha] \varphi \wedge(\text { Ufs } U i[M \neg f \alpha] \varphi) \\
& \equiv @[M f \alpha] \varphi
\end{array}
$$

Here, the abbreviation @Mf $\alpha$ designates the action $M f \alpha$ as an aborted goal. 


\section{The user asks the machine to inform something}

In this case (a question $U f f s$ ), the goal has the same evolution that in the previous one, but the machine response is of the type $M f s$. It is necessary to remember that ffs is a short form for $f f$ share, where to share is a base action.

$$
\begin{aligned}
& {[U f f s \phi](M s \text { Ui }[M f s \phi])} \\
& \equiv[\text { Uffs } \phi](?[M f s \phi] \varphi)
\end{aligned}
$$

Here the abbreviation ?Mfs $\phi$ designates the sharing of information $M f_{s} \phi$ as a proposed goal.

\section{The machine asks the user to inform something}

In this famework for human computer interaction, the machine has no intentions. However, it can take some initiative when the information required to complete a task is incomplete. Basically, the machine can generate a subdialog in order to request some complement ary information to the user. Different to a user request, the evolution of a subgoal proposed by the machine has just the following two stages.

i) A proposed subgoal is the effect of a machine request for complementary information to the user.

$$
[M f f s \phi](M s U i[U f s \phi] \varphi) \equiv[M f f s \phi(?[U f s \phi] \varphi)
$$

Here the abbreviation? $U f s \phi$ designates the action $U f s \phi$ as a proposed su boal.

ii) A reached, and consequently, satisfied subgoal materializes when the answer waited for the machine is true.

$$
\begin{array}{rl}
M s & U i[U f s \phi] \varphi \wedge[U f s \phi] \varphi \\
& \equiv ?[U f s \phi] \varphi \wedge[U f s \phi] \varphi \\
& \equiv++[U f s \phi] \varphi
\end{array}
$$

Here the abbreviation $++U f s \phi$ designates the action $U f s \phi$ as a satisfied subgoal.

iii) An aborted subgoal occurs when the user informs the machine that he will not answer the given request.

$$
\begin{aligned}
M s U i & {[U f s \phi] \varphi \wedge[U f s[U \neg f s \phi] \varphi] \gamma } \\
& \equiv ?\left[U f_{s} \phi\right] \varphi \wedge\left[U f_{s}\left[U \neg f_{s} \phi\right] \varphi\right] \gamma \\
& \equiv @[U f s \phi] \varphi
\end{aligned}
$$

Here, the abbreviation@Ufs $\phi$ designates the action Ufs $\phi$ as an aborted goal.

\subsubsection{Cooperative dialog}

As explained in the above sections, the cooperative dialog is produced around a task, where the machine is just a collaborator in it achievement. In our case, the machine has the obligation to resolve the proposed goal, for that it will be necessary its spoken intervention when lack the information to complete the task. The following axioms describe the cooperative dialog under these considerations. 
The machine is obligated to reach the proposed goal

If the user orders the realization of a task, and the machine knows the plan Msf, knows how to do it), then the machine executes this plan

(A5.i) ? $[M f \alpha] \varphi \wedge M s f \alpha \wedge \alpha \in A b \supset[M f \alpha] \varphi$

for a base action (i.e. elementary instruction)

(A5.ii) ? $\left[M f\left(\beta_{1} ; \beta_{2}\right)\right]\left(\varphi_{1} \wedge \varphi_{2}\right) \wedge M s f \beta_{l} \supset\left[M f \beta_{l}\right]\left(\varphi_{1} \wedge\left[U f f \beta_{2}\right]\left(?\left[M f \beta_{2}\right] \varphi_{2}\right)\right)$

for a complex task (i.e. a sequence of basic actions)

\section{The ignorance of the machine generates a question}

In this case, the machine knows the proposed task, but requires more information to accomplish it. Consequently, the task must be stopped until the information is completed. The machine executes two complementary actions before the tasks: (1) it asks the user about the required information, and (2) it verifies the user answer.

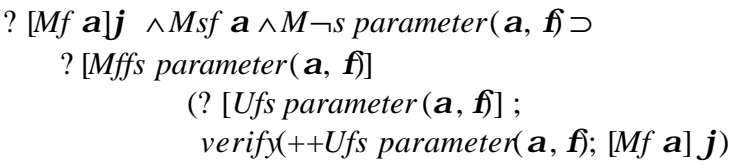

\section{A brief example}

The following example shows the structure of a short dialog where the interchanges of information converge to a goal in a world of design. For this example, we take a small fragment of a dialog from the DIME corpus [18]. This corpus was constructed for studying the multimodal interaction in the domain of kitchen design. In the selected fragment the user asks the system to reallocate the kitchen sink. How ever, he does not specify the new position, causing a subdialog (see step 4) by the system in order to obtain such information.

\section{The knowledge of the system at this moment:}

(C1) Msf move(Obj, NewLocation) The machine knows the base action to move

utt259: u: now move the kitchen sink (ahora a recorrer el fregadero) [Uff move(obj53, ?)]

1. $[$ Uff move(obj53,?)]( ? Mf move(obj53,?))

$$
\text { Definition of } U f f
$$

the proposed goal

2. ? [Mffsparameter (move(obj53,?),?)]

(? [Ufs parameter (move(obj53,?), ?)];

verify $(++U f$ s parameter (move(obj53, ?), ?);

Mf move(obj53, ?))

$1, \mathrm{C} 1, \mathrm{~A} 6$

a generated subgoal 
3. [Mffs parameter (move (obj53,?), ?)]

(? [Ufs parameter (move (obj53,?), ?)];

verify $(++U f$ s parameter (move(obj53, ?), ?);

Mf move(obj53, ?) )

2, A5.i

[Mffs parameter(move(obj53, ?), ?)]

utt260: $s$ : where do you want it? (¿a dónde quieres que lo ponga?)

4. (? [Ufs parameter (move(obj53, ?), ?)];

verify $(++U f$ s parameter (move(obj53, ?), ?);

Mf move(obj53, ?) )

Definition of $M f f s$

proposed subgoal

utt261: $u$ : move it to the dishwasher (recorrerlo hacia la máquina lava trastes) [Ufs parameter (move (obj53, loc45), loc45)]

5. [Ufs parameter(move(obj53, loc45), loc45)]

( Ms parameter(move (obj53, loc45), loc45)

Definition of $U f s$

6. ++ Ufs parameter(move(obj53, loc45), loc45)

4,5

satisfied subgoal

7. $[M f$ move(obj53, loc45)](hold(location (obj53, loc45)))

3,6 , and the definition of move

[Mf move (obj53, loc45)]

$\mathrm{M}$ : <reallocation of the kitchen sink to the new position in the graphical context>

8. + + Mf move $($ obj53, loc45)]

1,7

reached goal

\section{Conclusions}

This paper establishes the basis of a multimodal dialog model. In this model, the user activities are characterized by his goals, which at the same time give a structure to the dialog.

The proposed dialog model is based on a modal logic framework. This logic framework considers the action its central element. Thus, a spoken intervention is contemplated just as another form of action. This way, the dialog conduces the execution of an action, and the action causes the dialog.

In addition, our logic framework describes the dialog (i.e., the interchange of information) as the evolution of the goal proposed by the user. This evolution is a sequence of spoken and direct actions defined by the user intentions and the machine knowledge.

As future work, we plan to define new axioms that allow describing other phenomenon of the multimodal conversations, such as the resolution of the incomprehension related with the problems of communication. 
Acknowledgements. This work has been partly supported by the CONACYT (project 31128-A), the Laboratorio Franco-Mexicano de Informática /LAFMI (project "Interaction Parlée Homme-Machine") and the Human Language Technologies Labor atory of INAOE.

\section{References}

1. Austin, J., How to Do Things with Words. Harvard University Press, 1962.

2. Caelen, J. Vers une logique dialogique. Séminaire International de Pragmatique, Jerusalem, 1995.

3. Cohen, P. R. \& Levesque, H.J. Persistence, Intention and Commitment. Intentions in Comm unication. (eds.) P. R. Cohen, J. Morgan \& M. E. Pollack. The MIT Press. 1990.

4. Cohen, P. R. et Levesque, H. J. Rational interaction as the basis for communication. In Intentions in Communication. P.R. Cohen, J. Morgan et M. Pollack. (eds.) MIT Press. 1990.

5. Cohen, P. R. et Perrault, C. R. Elements of a Plan-Based Theory of Speech Acts. Cognitive Science, 3(3) : 177-212, 1979.

6. Halpern, J.Y. \& Moses, Y. A guide to completeness and complexity for modal logics og knowledge and belief. Artificial Inteligence 54, 319-379. Elsevier Science Publi shers. 1992.

7. McTear, M. Spoken Dialogue Technology: Enabling the Conversational User Interface. ACM Computing Surveys, Vol.34, No. 1, March 2002, pp 90-169.

8. Sadek, D. Logical task modelling for man-machine dialogue. AAAI-90 Proceedings. Eighth National Conference on Artificial Intelligence. pp 970-5 vol.2

9. Oviatt, S.L. "Breaking the Robustness Barrier: Recent Progress on the Design of Robust Multimodal Systems," Advances in Computers (ed. by M. Zelkowitz), Academic Press, 2002, vol. 56, 305-341.

10. Prendinger, H. \& Schurz, G. Reasoning about Action and Change. A Dynamic Logic Approach. Journal of Logic, Language, and Information, 5:209-245, 1996.

11. Searle, J.R. Actes de Langage, Hermann ed., Paris, 1972.

12. Taylor, M., Néel, F. \& Bouwhuis, D. (eds.) The Structure Of Multimodal Dialogue II. John Benjamins Publishing Company. 2000.

13. van der Hoek, W., van Linder, B. \& Meyer, Ch. J.-J. A Logic of Capabilities (extended abstract). Proceedings of the Third International Symposium of Logic Foundations of Computer Science. LFCS, 94. (eds.) A. Nerode \& Yu. V. Matiyasevich. San. Petersburgo, Rusia, Julio 11-14, Springer- Verlag, 1994.

14. van der Hoek, W., van Linder, B. \& Meyer, Ch. J.-J. 1994. Unraveling Nondeterminism : On having the Ability to Choose (extended abstract). Proceedings of the sixth International Conference on Artificial Intelligence : Methodology, Systems, Applications AIMSA'94. (eds.) P. Jorrand \& V. Sgurev. Sofía, Bulgaria, Septiembre 21-24, 1994. World Scientific. 1994.

15. van Linder, B., van der Hoek, W. \& Meyer, Ch. J.-J. Communicating Rational Agents. Proceedings of the $18^{\text {h }}$ German Annual Conference on Artificial Intelligence KI-94 : Advances in Artificial Intelligence. (eds.) B. Nebel \& L. Dreschler-Fischer. Saarbrücken, Alemania, Septiembre 18-23, 1994. Springer-Verlag 1994.

16. Vanderveken, D. La logique illocutoire. Mandarga éd. Bruselas, 1990.

17. Vernant, D. Modèle projectif et structure actionnelle du dialogue informatif. Du dialogue, Recherches sur la philosophie du langage, Vrin éd., París, n¹4, p. 295-314, 1992.

18. Villaseñor, L., Massé, A. \& Pineda, L.A. The DIME corpus. ENC01, 3er Encuentro Internacional de Ciencias de la Computación, Aguascalientes, México, SMCC-INEGI 2001. 